\title{
OFF-LABEL UTILIZATION OF ANTIDEPRESSANTS
}

\author{
Daniele Fabio Zullino ${ }^{1^{*}}$, Bruno Schwartz ${ }^{2 *}$, Romain Bilancioni ${ }^{3}$, Pierre Baumann ${ }^{3}$ \\ University Hospitals of Geneva, Switzerland: Division of Substance Abuse ${ }^{1}$, Private Practice, Geneva, Switzerland ${ }^{2}$, \\ University Department of Psychiatry, Prilly-Lausanne, Switzerland ${ }^{3}$; "The contributions of these authors are equivalent
}

\begin{abstract}
Summary: While antidepressant prescription rules are established for approved indications by large-scale studies, off-label utilization naturally often lacks the validation by large scientific databases, and is at its best based on expert consensus. The aim of the present survey was to study the prescription habits of hospital psychiatrists with regard to antidepressants, comparing patients treated for depressions and anxiety disorder with patients receiving off-label antidepressant treatment. Methods: Data on drug use for this study were based on 6 reference days from April 1999 to November 2001 in the 98 bed psychiatric hospital of the University of Lausanne, Switzerland. The drug prescriptions of 174 patients were assessed. Results: Whereas the diagnosis did not influence the choice between newer or older antidepressants, patients presenting an anxiety disorder were 4.5 times more likely $(p<0.05)$ and patients with other diagnoses 8 times more likely $(p<0.001)$ to receive an antipsychotic comedication compared to patients whose primary diagnosis was a depressive disorder. Also, patients receiving concomitantly a nonbenzodiazepine hypnotic were less likely to be prescribed an older antidepressant $(p<0.05)$. While patients with anxiety disorder and those with major depression received their antidepressants at comparable doses, patients with an off-label indication were treated preferentially with lower doses. Conclusions: The results of this survey suggest, that the prescribing hospital psychiatrists developed preferences with regard to the choice of the antidepressant class, which they then used for both registered and off-label indications. They then seemed to adapt the dose and the comedication according to the diagnosis, confirming the initial study hypothesis.
\end{abstract}

Key words: Antidepressants; Prescription habits; Off-label use; Depression; Anxiety disorders

\section{Introduction}

The analysis of factors which influence the prescription of psychotropic drugs, and especially antidepressants, has repeatedly been the subject of recent research $(2,4)$. Prescription habits have been shown to be especially influenced by characteristics of the regional health care system, physician management style, physician specialty and training, public attitudes, drug cost and availability, patient preferences, local tradition, education, marketing and formulary $(5,7,8,13,19)$. Conversely, prescribing physicians seem to take less into account patient characteristics $(5,8)$.

A major source of concern of many drug utilization studies has been the appropriateness of psychotropic drug prescribing practices, pointing out the frequent lack of concordance between psychiatric diagnoses and prescribed psychotropic medications $(4,8,18)$.

While tricyclic antidepressants were rapidly used for other indications after their introduction, this broadening of indications has been even more of an issue for the newer generation antidepressants. Many of the newer drugs can presently be considered valid alternatives, especially in the treatment of anxiety disorders (3) and eating disorders (10).
Their popularity for the treatment of depressive symptoms in patients with somatic comorbidities is also largely due to their enhanced safety profiles, relative to those observed in older drugs (15). The broad range of diagnoses for which antidepressants are prescribed has recently been pointed out in some drug utilization surveys, where up to $40 \%$ of patients receiving an antidepressant were not diagnosed with depression $(14,16)$.

While guidelines for the use of antidepressant medications have been established for approved indications by several large-scale studies, the off-label utilization naturally often lacks this validation, and is at most based on expert consensus.

Whereas differences between patients treated with antidepressants for approved indications and those with offlabel prescriptions have been found with regard to the intended treatment duration (16), showing shorter treatment duration for off-label utilization, data comparing other aspects of antidepressant treatment, such as doses, comedications etc., is still lacking.

The aim of the present survey is to study the prescription habits of hospital psychiatrists with regard to antidepressants, comparing patients treated for depression and 
anxiety disorder with patients receiving off-label antidepressant treatment, examining particularly the administered doses and comedications. The underlying hypothesis was that physicians would choose the same class of antidepressants in off-label indications as they would for approved indications, but that they would adjust doses and comedication in response to the clinical situation.

\section{Methods}

The present study was developed as part of the AMSP project (Arzneimittelsicherheit in der Psychiatrie = drug safety in psychiatry), which is a program for continuous assessment of adverse drug reactions in psychiatric inpatients under naturalistic conditions of routine clinical treatment. The methodology has been described elsewhere $(6,21,23$, 24). Currently, more than 35 German, Swiss, Austrian, Hungarian and Belgian sites are participating. Data on drug use in the participating hospitals are based on two reference days per year. All drugs given on a reference day are recorded along with age, sex, and diagnosis (ICD-10) for all patients under surveillance. The daily dosage is also recorded.

Data of the present study were drawn out of 6 reference days from April 1999 to November 2001 in the 98-bed psychiatric hospital of the University of Lausanne, Switzerland. Currently, the mean hospitalization duration is 14 days and the nurse/bed ratio is 0.95 .

\section{Definition of drug classes}

Antidepressants were classified into tricyclic drugs (TCA) and drugs of the newer generations (DNG). DNGs were defined as the selective serotonin re-uptake inhibitors paroxetine, sertraline, fluoxetine, citalopram and fluvoxamine as well as mirtazapine, reboxetine, nefazodone and moclobemide. The class of TCAs included the tricyclic compounds clomipramine, trimipramine, imipramine, amitriptyline and dibenzepin.

To analyze the effect of prescribing patterns of sedating vs nonsedating antidepressants and concomitant antipsychotic medication or benzodiazepines, the antidepressant group was divided into sedating (SA) versus nonsedative drugs (NSA). The sedative compounds were: trimipramine, clomipramine, nefazodone, and mirtazapine.

Benzodiazepines were classified as one group, including sedative and hypnotic drugs, as sedative benzodiazepines were often also used as hypnotics, the different indications therefore being difficult to assess. Nonbenzodiazepine hypnotics $(\mathrm{NH})$ formed a further drug class, including zolpidem, zopiclone and zaleplon.

\section{Dose ranges}

In order to compare the antidepressant prescription with regard to applied doses, three dose ranges were defined for each administered drug (Tab. 1). For patients receiving more than one antidepressant, the doses were summed up: twice the low dose range giving a middle dose range, a low dose and a middle dose or two middle doses giving a high dose.

\section{Label and off-label indications}

Based on the primary diagnosis, patients were grouped into 3 classes: (1) Depression, (2) Anxiety Disorder, and (3) Other Disorders.

As no patient had a manic or mixed episode as primary diagnosis, all patients presenting an ICD-10 diagnosis of affective disorder were depressive.

Whereas all used antidepressants are approved in Switzerland for depression, they differ with regard to the approval for the different anxiety disorders. Anxiety disorders were nevertheless grouped into one class, as knowledge of the diverse approved indications among the prescribing physicians could not be assumed. On the other hand, physicians are locally trained to consider newer antidepressants as the first line pharmacotherapy for anxiety disorders.

\section{Analyses}

In descriptive data analyses means and standard deviations were calculated for numerical variables while nominal ones frequency categories values and percentages are reported. In exploratory analyses, the differences between groups were tested with chi-square tests (for nominal variables) and analyses of variance for numerical ones.

Predictive models were built with multivariate logistic regression analysis. Binary stepwise logistic regression analysis was used to determine factors predicting the prescription of older antidepressants, antipsychotics, benzodiazepines and nonbenzodiazepine hypnotics. A further model predicting the antidepressant dose range used was analyzed by multinominal stepwise logistic regression. Multinominal logistic regression broke the regression up into a series of binary regressions comparing each group to a baseline group, which we determined to be the low dose range group. The backward Wald method was applied.

Tab. 1: Dose ranges (in $\mathrm{mg} / \mathrm{d}$ ) of antidepressants prescribed in this study.

\begin{tabular}{|l|c|c|c|}
\hline & Low & Medium & High \\
\hline Citalopram & $<40$ & $40-60$ & $>60$ \\
\hline Fluoxetine & $<40$ & $40-60$ & $>60$ \\
\hline Paroxetine & $<40$ & $40-60$ & $>60$ \\
\hline Fluvoxamine & $<100$ & $100-200$ & $>200$ \\
\hline Sertraline & $<100$ & $100-200$ & $>200$ \\
\hline Venlafaxine & $<150$ & $150-300$ & $>300$ \\
\hline Nefazodone & $<200$ & $200-400$ & $>400$ \\
\hline Reboxetine & $<4$ & $4-8$ & $>8$ \\
\hline Mirtazapine & $<30$ & $30-60$ & $>60$ \\
\hline Trimipramine & $<100$ & $100-200$ & $>200$ \\
\hline Clomipramine & $<150$ & $150-300$ & $>300$ \\
\hline Imipramine & $<150$ & $150-300$ & $>300$ \\
\hline Amitriptyline & $<150$ & $150-300$ & $>300$ \\
\hline Dibenzepin & $<160$ & $160-360$ & $>360$ \\
\hline Moclobemide & $<300$ & $300-600$ & $>600$ \\
\hline
\end{tabular}


The data were analyzed using the SPSS for Windows program, version 12.0 .

\section{Results}

\section{Characteristics of the sample}

The drug prescriptions of 174 patients were assessed. The mean age was $42.6 \pm 11.6$ (range 20-66) and the proportion of women $59.2 \%$. There were no differences between index days with regard to age and sex distribution.

The distribution regarding the primary ICD-10 diagnosis was: Mental and behavioral disorders due to psychoactive substance use (F10): 10 (5.7 \%); Schizophrenia, schizotypal and delusional disorders (F20): 47 (27.0\%); Mood disorders (F30): 81 (46.6\%); Neurotic, stress-related and somatoform disorders (F40): 10 (5.7 \%); Behavioral syndromes associated with physiological disturbances and physical factors (F50): 1 (0.6 \%); Disorders of adult personality and behavior (F60): 25 (14.4\%).

\section{Number of prescribed drugs per patient and comedications}

The mean number of drugs administered was $4.3 \pm 1.7$ (range 1-10). Forty-eight patients received nonbenzodiazepine hypnotics (27.6 \%), 138 (79.3\%) had benzodiazepines prescribed, 25 (14.0\%) received conventional antipsychotics, 61 (35\%) atypical antipsychotics, 22 (12.6\%) anticonvulsants, 13 (7.5\%) lithium, and 104 (59.8\%) somatic drugs. There was no secular effect with regard to the number of prescribed drugs and comedications when comparing the prescriptions of 1999, 2000 and 2001.

\section{Type of antidepressants}

Among the investigated patients, 102 (58.6\%) were treated with SSRIs, 18 (10.3\%) patients received venlafaxine, $22(12.6 \%)$ received mirtazapine, 29 (16.7\%) had a tricyclic antidepressant (TCAs), and 16 (9.2\%) received another drug. Thirteen patients $(7.5 \%)$ were treated simultaneously with two antidepressants

\section{Impact of patients characteristics on antidepressant presc- ription}

The prescription of the different types of antidepressants for patients with depression, anxiety disorder and others are shown in Tab. 2.

The proportion of patients receiving TCA were $16 / 81$ (19.8\%) for depressive patients, 2/10 (20\%) for patients presenting an anxiety disorder, and 11/83 (13.3\%) among the patients with another disorder (Chi-square 1.322; n.s.).

Table 3 shows the result of the stepwise logistic regression, with the choice of an older antidepressant being the dependent variable and the index year, the diagnostic class, sex, age and the prescription of concomitant drugs (benzodiazepines, nonbenzodiazepine hypnotics, anticonvulsants, lithium, somatic drugs) as independent parameters. With increasing age the probability of receiving an older antidepressant increased $(p<0.05)$. Furthermore, a clear secular trend appeared, as the probability of receiving an older antidepressant was significantly lower in $2000(\mathrm{p}<0.05)$ and in 2001 ( $\mathrm{p}<0.05)$ compared with 1999. Also, patients receiving concomitantly a nonbenzodiazepine hypnotic were less likely to be prescribed an older antidepressant $(p<0.05)$. The diagnostic class was not retained as a predictive parameter. The positive predictive value of the model was $85.1 \%$.

\section{Prescription of concomitant antipsychotic drugs}

The correspondent result of the stepwise logistic regression analysis is shown in Tab. 4. The following parameters were entered: Index year, sex, age, diagnostic class, antidepressant type (older vs. newer) and concomitant treatments. Only the diagnostic class was retained, patients with anxiety disorder or other non-depressive disorders presenting significantly higher odds-ratios compared to patients with the diagnosis of depression. The positive predictive value of the definitive model was $73.0 \%$.

Tab. 2: Diagnostic class and type of prescribed antidepressant.

\begin{tabular}{|l|r|r|c|r|r|r|}
\hline & \multicolumn{2}{|l|}{ Depression } & \multicolumn{2}{|c|}{$\begin{array}{c}\text { Disorder } \\
\text { Anxiety }\end{array}$} & \multicolumn{2}{c|}{ Others } \\
\hline & $\mathrm{n}$ & $\%$ & $\mathrm{n}$ & $\%$ & $\mathrm{n}$ & $\%$ \\
\hline SSRI & 34 & 42.0 & 8 & 80.0 & 51 & 61.4 \\
\hline venlafaxine & 11 & 13.6 & 0 & 0.0 & 4 & 4.8 \\
\hline mirtazapine & 12 & 14.8 & 0 & 0.0 & 8 & 9.6 \\
\hline tca & 6 & 7.4 & 1 & 10.0 & 0 & 12.0 \\
\hline others & 7 & 8.6 & 0 & 0.0 & 9 & 10.8 \\
\hline combination & 11 & 13.6 & 1 & 10.0 & 1 & 1.2 \\
\hline
\end{tabular}

Tab. 3: Stepwise logistic regression model for use of older antidepressants.

\begin{tabular}{|ll|c|c|c|}
\hline & OR $^{\mathrm{a}}$ & $\begin{array}{c}95 \% \text { Confidence } \\
\text { Intervals }\end{array}$ & $\mathrm{p}$ \\
\hline Age & 1.05 & $1.01-1.09$ & 0.015 \\
\hline Index year 1999 & & & 0.011 \\
& 2000 & 0.24 & $0.08-0.72$ & 0.011 \\
& 2001 & 0.29 & $0.10-0.84$ & 0.022 \\
\hline $\begin{array}{l}\text { Nonbenzodiazepine } \\
\text { hypnotic }\end{array}$ & 0.25 & $0.07-0.92$ & 0.037 \\
\hline
\end{tabular}

${ }^{a}$ Odds ration for the probability of receiving an older antidepressant

Tab. 4: Stepwise logistic regression model for use of concomitant antipsychotic drugs.

\begin{tabular}{|l|c|c|c|}
\hline $\begin{array}{l}\text { Diagnostic } \\
\text { class }\end{array}$ & $\mathrm{OR}^{\mathrm{a}}$ & $\begin{array}{c}95 \% \text { Confidence } \\
\text { Intervals }\end{array}$ & $\mathrm{p}$ \\
\hline Depression & 1 & & $<0.001$ \\
\hline Anxiety & 4.51 & $1.13-17.94$ & 0.033 \\
\hline Other & 8.59 & $4.19-17.60$ & $<0.001$ \\
\hline
\end{tabular}

${ }^{\mathrm{a}}$ Odds ration for the probability of receiving a concomitant antipsychotic drug 


\section{Prescription of concomitant benzodiazepines}

A stepwise logistic regression predicting the prescription of benzodiazepines including the following parameters was computed: Sex, index year, age, diagnostic class, antidepressant type (older vs. newer and sedative vs. non-sedative), and concomitant treatments. None of the parameters was retained.

\section{Prescription of concomitant nonbenzodiazepine hypnotics}

The following parameters were used to compute the corresponding stepwise logistic regression (Tab. 5): Sex, index year, age, diagnostic class, antidepressant type (older vs. newer and sedative vs. non-sedative), and concomitant treatments. Only the parameter comparing older vs. newer antidepressants was retained. Patients receiving an older antidepressant had a lower probability of receiving a concomitant nonbenzodiazepine hypnotic $(p<0.05)$. The positive predictive value of the retained model was $72.4 \%$.

\section{Dose range}

The distribution with regard to the prescribed doses was: Low dose 48 patients (27.6\%), medium dose 96 (55.2\%) and high dose 30 (17.2\%). The multinominal stepwise logistic regression analysis was computed with the following parameters: Sex, diagnostic class, antidepressant class (newer vs. older), and prescription of antipsychotics, benzodiazepines, nonbenzodiazepine hypnotics, anticonvulsants, lithium or somatic drugs (Tab. 6). The group of individuals having received the antidepressant medication at a low dose range were defined as the reference group. The parameters of the diagnostic class and of the antidepressant class were retained. Whereas patients with anxiety disorder

Tab. 5: Stepwise logistic regression model for use of concomitant nonbenzodiazepine hypnotic.

\begin{tabular}{|c|c|c|c|}
\hline & OR & $\begin{array}{l}\text { 95\% Confidence } \\
\text { Intervals }^{\mathrm{a}}\end{array}$ & $\mathrm{p}$ \\
\hline $\begin{array}{l}\text { Prescription of an } \\
\text { older antidepressant }\end{array}$ & 0.26 & $0.07-0.89$ & 0.032 \\
\hline
\end{tabular}

${ }^{a}$ Odds ration for the probability of receiving a concomitant nonbenzodiazepine hypnotic did not differ from those with depressive disorder with regard to the applied doses, those with other diagnoses received less often medium $(p<0.001)$ and higher $(p<0.001)$ antidepressant doses. Furthermore, those receiving an older antidepressant had an odds ratio of 6.66 to receive their antidepressant treatment rather at a high than at low dose, compared to the patients having a treatment with newer antidepressants only $(\mathrm{p}<0.01)$.

\section{Discussion}

The aim of the present study was to investigate if hospital psychiatrists would prescribe antidepressants and comedication differently for patients with a registered indication compared to those administered antidepressants on an offlabel basis.

Whereas the diagnosis did not seem to influence the choice of a newer or and older antidepressant, it was significantly associated with the prescription of antipsychotic comedication and antidepressant dose. Patients presenting an anxiety disorder were 4.5 times more likely and patients with other diagnoses 8 times more likely to receive an antipsychotic comedication compared to patients whose primary diagnosis was a depressive disorder. While patients with anxiety disorder and those with major depression received their antidepressants at comparable doses, patients with an off-label indication were treated preferentially with lower doses.

While antipsychotics, on one hand, high potency as well as low potency, have often been advocated as being valid alternatives to benzodiazepines as anxiolytic treatments, and, on the other hand, sedative antidepressants have been suggested to reduce the need of concomitant benzodiazepine use, our data did not confirm these assumptions. Neither the use of sedative antidepressants, nor the prescription of benzodiazepines had an impact on the prescription of concomitant antipsychotics. Antipsychotics were only prescribed in the function of the diagnosis.

A secular trend was found with regard to the prescription of tricyclic antidepressants, these older drugs being less likely to be prescribed in 2000 and 2001 compared to 1999 , even when checking for confounding factors like diagnosis or comedication. As the prescription of a nonbenzodiaze-

Tab. 6: Logistic regression model for choice of antipsychotic dose range.

\begin{tabular}{|c|c|c|c|c|c|c|}
\hline & \multicolumn{3}{|c|}{ Medium dosis } & \multicolumn{3}{c|}{ High dosis } \\
\hline & OR & $\mathrm{p}$ & $\begin{array}{c}\text { 95\% Confidence } \\
\text { Intervals }\end{array}$ & OR & $\mathrm{p}$ & $\begin{array}{c}95 \% \text { Confidence } \\
\text { Intervals }\end{array}$ \\
\hline Diagnostic class & & & & & & \\
\hline Depression & & & & & & \\
\hline Anxiety & 0.98 & 0.988 & $0.10-9.35$ & 2.21 & 0.514 & $0.21-23.83$ \\
\hline Other & 0.21 & $<0.001$ & $0.09-0.48$ & 0.08 & $<0.001$ & $0.03-0.27$ \\
\hline $\begin{array}{l}\text { Prescription of an } \\
\text { older antidepressant }\end{array}$ & 1.11 & 0.865 & $0.34-3.56$ & 6.66 & 0.004 & $1.82-24.29$ \\
\hline
\end{tabular}


pine hypnotic was associated with a lowered odds of receiving an older antidepressant, one could hypothesize that the observed secular trend may be due to the replacement of tricyclics used for their sedative or sleep-enhancing properties by nonbenzodiazepine hypnotics.

As in previous studies investigating prescription habits of psychotropic drugs $(1,9,11,12,17)$, polypharmacy was highly prevalent, the mean number of prescribed drugs being 4 with a range from 1 to 10 drugs per patient. Whereas polypharmacy has long been considered a malpractice in earlier studies, it has become increasingly apparent nowadays that psychiatric polypharmacy can have some advantages, i.e., to further improve sleep, have a more potent anxiolytic or sedative effect and to overcome therapy resistances $(17,20,22)$.

One further intriguing finding was the observation that the risk of receiving a tricyclic antidepressant augmented with increasing age. This is at first sight counter-intuitive, as the risk of cardiovascular and other side effects may be more important in elder patients and should have discouraged the doctors from prescribing these drugs for those patients. On the other hand, elder patients may be often the more chronic patients and may have received the antidepressant for longer habitual treatment, which the physician could possibly not have had the courage to change anymore. As the present data are only collected on a crossover basis, this assumption can, however, not be tested out.

The results of this study need to be viewed against their methodological limitations. The method of index day assessments allows only cross sectional analyses for each patient. The course of the prescription, i.e., titration schemes, and the duration of the antidepressant treatment as well as of the comedications cannot be evaluated. That not every patient can be considered as having been already stabilized on the medication at the index day, especially data on doses, but also all other data, has to be interpreted with caution. Also, diagnoses were not based on a structured interview, which may have diminished their validity. However, as the ICD-10 diagnoses were derived post hoc from the medical records, they surely better reflect clinical practice. Furthermore, only the first diagnosis (hospitalization indication) was retained.

The two reference days were always fixed for the period of late spring and late autumn, allowing each center to choose a day within \pm 7 days. The choice of seasonally fixed reference days may be a confounding factor in depressive disorders as well as anxiety disorders.

With regard to generalizability, one has to consider that the survey was limited to one site only, the hospital being University based and organized in specialized wards. The refuse, biases by local customs and conditions of practice cannot be excluded.

In conclusion, the present results thus suggest that the prescribing hospital psychiatrists developed preferences with regard to the choice of antidepressant class, which they then used for both registered and off-label indications. They then seemed to adjust the dose and the comedication according to the diagnosis, confirming the initial study hypothesis.

Factors influencing this spreading out of prescription habits from labeled to off-label indications remain to be explored. The results furthermore generate various related important questions, which should be addressed through further research: Does the rapid spreading out of off-label prescription increase or decrease the risk of adverse drug reactions? Does it unduly increase or decrease costs? What is the impact of doctors' interaction with pharmaceutical companies?

\section{References}

1. Ambühl B, Würmle O, Michel G. Die Verschreibungspraxis von Psychopharmaka in einer psychiatrischen Unversitätsklinik. Psychiatr. Prax. 1993;20:70-73.

2. Bingefors $\mathrm{K}$, Isacson $\mathrm{D}$, von Knorring L. Antidepressant dose patterns in Swedish clinical practice. International Clinical Psychopharmacology 1997;12: 283-290.

3. Boerner RJ, Müller HJ. The importance of newer antidepressants in the treatment of anxiety/depressive disorders. Pharmacopsychiatry 1999;32:119-126.

4. Bouhassira M, Allicar MP, Blachier C, Nouveau A, Rouillon F. Which patients receive antidepressants? A 'real world' telephone study. Journal of Affective Disorders 1998;49:19-26.

5. Egberts ACG, Veenstra M, de Jong-van den Berg LTW. Antidepressant drug choice for first users in two regions in the Netherlands. Pharm. World Sci. 1999; 21:132-136.

6. Grohmann R, Rüther E, Engel RR, Hippius H. Assessment of adverse drug reactions in psychiatric inpatients with the AMSP drug safety program: methods and first results for tricyclic antidepressants and SSRI. Pharmacopsychiatry 1999;32:21-28

7. Laux G, Baier D. Quality-monitoring of psychotropic drug therapy in post-marketing surveillance. Pharmacopsychiatry 1997;30(suppl):21-27.

8. Linden M, Lecrubier Y, Bellantuono C, Benkert O, Kisely S, Simon G. The prescribing of psychotropic drugs by primary care physicians: an international collaborative study. Journal of Clinical Psychopharmacology 1999;19:132-140.

9. Lippert E, Aigner JM, Grohmann R, Klein HE, Schmauss M, Rüther E. Anwendungshäufigkeiten und Dosierungen von Psychopharmaka an psychiatrischen Versorgungskrankenhäusern - Ergebnisse aus dem Arzneimittelüberwachungsprojekt Bayern. Psychopharmakotherapie 1996;3:178-183.

10. Mayer LES, Walsh BT. The use of selective serotonin reuptake inhibitors in eating disorders. Journal of Clinical Psychiatry 1998;59(suppl 15):28-34

11. Morgan R, Gopalaswamy AK. Psychotropic drugs: another survey of prescribing patterns. Br.J.Psychiatry 1984;144:298-302.

12. Muijen M, Silverstone T. A comparative hospital survey of psychotropic drug prescribing. Br.J.Psychiatry 1987;150:501-504.

13. Olfson M, Pincus HA, Dial TH. Professional practice patterns of U.S. psychiatrists. American Journal of Psychiatry 1994;151:89-95.

14. Ornstein S, Stuart G, Jenkins R. Depression diagnoses and antidepressant use in primary care practices: a study from the Practice Partner Research Network (PPRNet). J Fam.Pract. 2000;49:68-72.

15. Pincus HA, Tanielian TL, Marcus SC, Olfson M, Zarin DA, Thompson J, et al. Prescribing trend in psychotropic medications: primary care, psychiatry, and other medical specialities. JFMA 1998;279:526-531.

16. Pomerantz JM, Finkelstein SN, Berndt ER, Poret AW, Walker LE, Alber RC, et al. Prescriber intent, off-label usage, and early discontinuation of antidepressants: a retrospective physician survey and data analysis. J Clin.Psychiatry 2004;65: 395-404.

17. Rittmansberger H, Meise U, Schauflinger K, Horvath E, Donat H, Hinterhube H. Polypharmacy in psychiatric treatment. Patterns of psychotropic drug use in Austrian psychiatric clinics. Eur.Psychiatry 1999;14:33-40.

18. Tinsley JA, Shadid GE, Li H, Offord KP, Agerter DC. Psychotropic prescribing practices and educational needs: a survey of family physicians and psychiatrists. Gen.Hosp.Psychiat. 1998;20:360-367.

19. Tylee A. Depression in the community: physician and patient perspective. Journal of Clinical Psychiatry 1999;60(suppl 7):12-16.

20. Zullino D, Baumann P. Lithium augmentation in depressive patients not responding to selective serotonin reuptake inhibitors. Pharmacopsychiatry 2001;34: $1-9$. 
21. Zullino D, Baumann P, Grohmann R, Greil W. L'importance de la pharmacovigilance en psychiatrie - Le Projet AMSP (Arzneimittelsicherheit in der Psychiatrie). Revue médicale de la Suisse Romande 2000;120:105-109.

22. Zullino D, Bondolfi G, Baumann P. The serotonin paradox: negative symptom and SSRI augmentation. Int. J. Psych. Clin. Prac. 1998;2:19-26.

23. Zullino DF, Horvath A, Greil W, Grohmann R, Hippius H, Eich P, et al. Die Be- deutung der Pharmakovigilanz in der Psychiatrie: das AMSP-Projekt (Arzneimittelsicherheit in der Psychiatrie). Schw. Arch. Neurol. Psychiatr. 2002; 153:266-271.

24. Zullino D, Mayland G, Schmidt LG, Fahndrich E, Greil W, Horvath A, et a Prescribing practices in German and Swiss psychiatric university and in non-university hospitals: national differences. Int J Clin Pharmacol Ther 2005;43: $339-49$

Submitted March 2007. Accepted March 2008

\section{Corresponding author:}

Dr. Daniele Zullino, Service d'abus de substances, Hôpitaux Universitaires de Geneve Rue Verte 2, 1205 Geneve, Switzerland, e-mail: daniele.zullino@hcuge.ch 